PACS 72.20.Fr, 72.80.Ey, 73.20.At, 73.21.Fg, 73.63.Hs, 81.07.St

\title{
Electron mobility in the GaAs/InGaAs/GaAs quantum wells
}

\author{
V.V. Vainberg ${ }^{1}$, A.S. Pylypchuk ${ }^{1}$, N.V. Baidus ${ }^{2}$ and B.N. Zvonkov ${ }^{2}$ \\ ${ }^{1}$ Institute of Physics, National Academy of Sciences of Ukraine, 03680 Kiev, Ukraine \\ Phone: +38(044) 525-79-51, e-mail:vainberg@iop.kiev.ua \\ ${ }^{2}$ Research Scientific Physical-Technical Institute of Lobachevskii State University, 603950 Nizhni Novgorod, Russia
}

\begin{abstract}
The temperature dependence of the electron lateral mobility in quantum wells of the GaAs/InGaAs/GaAs heterostructures with delta-like doping has been studied. Two types of sample doping - in the quantum well and in the adjacent barrier at a small distance from the well - were used. In the case of shallow wells, in such structures the experimental results may be well described by known electron scattering mechanisms taking into account the shape of real envelope wave functions and band bending due to non-uniform distribution of the positive and negative space charges along the growth direction of heterostructure layers. In the case of delta-like doping in the well, a good agreement between experiment and calculations is achieved, if one takes into account a contribution to electron transport of the states of the impurity band formed by the deltaimpurity beneath the bottom of the lowest quantum subband.
\end{abstract}

Keywords: heterostructure, quantum well, electron mobility, lateral transport, semiconductor.

Manuscript received 15.01.13; revised version received 27.02.13; accepted for publication 19.03.13; published online 25.06.13.

\section{Introduction}

The GaAs/InGaAs/GaAs heterostructures with quantum wells (QW) are successfully applied in modern electronics. In particular, such structures enabled to create low-noise UHF transistors HEMT [1] and powerful laser diodes [2]. In the recent decade, the possibility to generate or detect the middle and far infrared radiation by using these structures was explored [3]. The latter is based on the real-space transfer of electrons under heating electric fields. In particular, the delta-doped structures with two tunnel-coupled quantum wells are used for this purpose. The current-voltage characteristics and accompanying far IR radiation of such structures in the regime of the lateral transport under strong electric fields within the temperature range 4 to $100 \mathrm{~K}$ were studied in [4]. In that paper, the electron mobility and real-space redistribution of electrons between wells as functions of electron temperature were calculated in the frame of a simple model of rectangular quantum wells. The obtained dependences predicted considerably higher redistribution and, consequently, a stronger dependence of the mobility and IR radiation on the electric field as compared to the experimental ones. The successive measurements for other structures did not eliminate this discrepancy.

For further studies of effects caused by the realspace transfer of electrons in these structures, it is important to investigate temperature and field dependences of the electron mobility in the quantum wells and analyze them with account of both the band bending and real envelope wave functions [5].

The mobility of carriers in quantum wells is sufficiently well studied in the case when the doping impurity is introduced only into the barrier and is separated from the well by a spacer. In these samples, the highest mobility, especially at low temperatures, is achieved. To generate IR radiation along with a high mobility in one of the quantum wells, it is important to have a low mobility in another one of the couple. It 
Table 1. Parameters of samples under study.

\begin{tabular}{|l|c|c|c|c|c|c|c|}
\hline Sample \# & $\begin{array}{c}\text { QW } \\
\text { width, } \AA\end{array}$ & $\begin{array}{c}\text { Barrier } \\
\text { width, } \AA\end{array}$ & $\begin{array}{c}\text { QW depth, } \\
\mathrm{meV}\end{array}$ & $\begin{array}{c}\text { Impurity } \\
\text { concentration, } \\
10^{11} \mathrm{~cm}^{-2}\end{array}$ & $\begin{array}{c}\text { Impurity } \\
\text { position }\end{array}$ & $\begin{array}{c}\text { Number of } \\
\text { periods }\end{array}$ & $\begin{array}{c}n_{4.2 \mathrm{~K}}, \\
10^{11} \mathrm{~cm}^{-2}\end{array}$ \\
\hline 5997 & 80 & 800 & 63 & 8.1 & QW centre & 10 & 7.85 \\
\hline 5998 & 80 & 800 & 63 & 2 & QW centre & 10 & 1.7 \\
\hline 6002 & 80 & 800 & 63 & 4.5 & QW centre & 10 & 4.05 \\
\hline 6215 & 80 & 800 & 96 & 6.1 & $\begin{array}{c}\text { barrier, } 100 \AA \\
\text { from QW }\end{array}$ & 10 & 4.6 \\
\hline 6291 & 100 & 800 & 50 & 2.1 & $\begin{array}{c}\text { barrier, } 10 \AA \\
\text { from QW }\end{array}$ & 20 & 1.6 \\
\hline
\end{tabular}

could be achieved by inserting a delta layer of impurities in the plane of the well. We do not know publications concerning systematic investigations of mobility in such samples, except of a few measurements for the systems of $\mathrm{AlGaAs} / \mathrm{GaAs} / \mathrm{AlGaAs}$ [6], InAlAs/InGaAs/InAlAs $[7,8] \mathrm{GaAs} / \mathrm{InGaAs} / \mathrm{GaAs}$ [9]. Therefore, the purpose of this work was to investigate temperature dependences of the conductivity and Hall mobility of electrons within the range 4 to $400 \mathrm{~K}$ in the $\mathrm{GaAs} / \mathrm{InGaAs} / \mathrm{GaAs}$ quantum wells with different concentrations of deltadoping impurity in the well plane. For the purpose of comparison, the structures with quantum wells deltadoped in adjacent barriers had been also investigated. The results of studying the field dependence of the electron mobility will be published later.

\section{Samples, experimental details and measurement results}

The GaAs/InGaAs/GaAs heterostructures were grown by the MOVPE method on semi-insulating GaAs (100) substrates. The structures were delta-doped by Si. All measurements were carried out with the samples listed in Table 1 with parameters following from the technological process data. The residual shallow impurity concentration in these structures is estimated to be within the limits of $10^{15}$ to $10^{16} \mathrm{~cm}^{-3}$. To compensate the influence of the surface states on the potential profile, the structures were doped by an impurity deltalayer near the surface. The samples for measurements of resistivity and the Hall effect were cut from wafers in the shape of the Hall bridge. The ohmic contacts were made by deposition of the $\mathrm{Pd} / \mathrm{Ge} / \mathrm{Au}$ layers in vacuum and further heating in the hydrogen ambient at $700 \mathrm{~K}$. Electric field was applied along $\langle 110\rangle$.

Measurements of the resistivity and Hall effect in the weak magnetic field $(B=0.2 \mathrm{~T})$ were carried out within the range 4 to $400 \mathrm{~K}$ in the $\mathrm{DC}$ regime $(I=100 \mu \mathrm{A})$. The applied electric field was less than $1 \mathrm{~V} / \mathrm{cm}$ for all the studied samples. The error in temperature measurements was no more than $0.1 \mathrm{~K}$. Measurements of the Hall effect were repeated after several months, and results for the same sample coincided with the accuracy within $1 \%$. At the same time, for different samples cut from one wafer, the Hall concentration may differ considerably, while their temperature dependences of mobility and Hall coefficient coincide qualitatively quite well.

The temperature dependences of the Hall coefficient $R_{\mathrm{H}}$ for all 5 structures under study are presented in Fig. 1 . These curves show comparatively small variation of the Hall coefficient over the whole temperature range. The observed behaviour may be caused both by variation of the charge carrier concentration and variation of the Hall factor. Typical for all curves initial growth of $R_{\mathrm{H}}$ with $T$ at low temperatures is related, from our point of view, with the increase of the Hall factor, because a carrier concentration decrease is unlikely in this temperature range ${ }^{1}$. On the other hand, the further decrease of $R_{\mathrm{H}}$ at least at temperatures higher than $100 \mathrm{~K}$ is caused by increasing the concentration of electrons with temperature growth. Indeed, since in all the structures at temperatures from 4 up to $10 \mathrm{~K}$ the electron gas is close to quantum degeneracy, the Hall factor at these temperatures must be in fact equal to unity ${ }^{2}$. Further, the high temperature Hall coefficient values are less than those at $4 \mathrm{~K}$. Hence, the Hall factor should be considerably less than unity, if the concentration is supposed to be constant with the growing temperature. However, this is impossible. Listed in the rightmost column of Table 1 are values of the impurity concentration in the samples at $4 \mathrm{~K}$ calculated from data presented in Fig. 1 when supposing that the Hall factor is equal to unity.

The temperature dependences of the conductivity for the samples with delta-doping in an adjacent barrier and in QW are shown in Figs $2 \mathrm{a}$ and $2 \mathrm{~b}$, respectively. The corresponding dependences of the Hall mobility $\mu_{H}=R_{H} \sigma$ on temperature calculated for these structures from the data shown in Figs $1,2 \mathrm{a}$ and $2 \mathrm{~b}$ are shown in Figs $2 \mathrm{c}$ and $2 \mathrm{~d}$.

\footnotetext{
${ }^{1}$ In principle, such a decrease may be related with capture of electrons by very shallow traps.

${ }^{2}$ Strictly, this statement is valid only in the case of common relaxation time for all charge carriers. For example, in the case of filling several subbands it may be violated.
} 


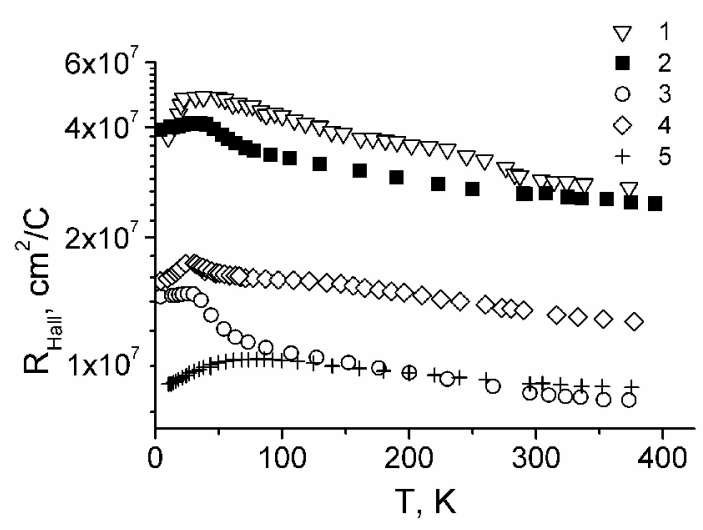

Fig. 1. Temperature dependences of the Hall coefficient for the samples under study. Sample \#\#: 1 - 5998, 2 - 6291, 3 - 6215, $4-6002,5-5997$.

Comparing data in Fig. 2, one may conclude that, as expected, the electron mobility and corresponding conductivity at low temperatures are considerably larger for samples doped in the barrier as compared to those doped in QW. The temperature dependences both for conductivity and mobility differ from each other substantially in these two cases. In the case of doping in the barrier, the electron mobility at first slightly increases with growth of temperature from $4 \mathrm{~K}$ and then strongly decreases. For the samples doped in the QW, it increases by 4 to 5 times, exhibits a flat maximum and slightly decreases with heating up to the room temperature. Furthermore, one should notice the following feature of the curves for the electron mobility in the samples doped in QW (Fig. 2d). At low temperatures (below 70-80 K) the higher is the impurity concentration, the higher is the mobility. For the sample with the lowest impurity concentration (approximately $2 \cdot 10^{11} \mathrm{~cm}^{-2}$, sample \#5998) the mobility value at $4.2 \mathrm{~K}$ differs from those in other samples by several times. At the same time, at higher temperatures the higher is the concentration, the lower is the mobility.

These results may be explained qualitatively as follows. As it is well known, in delta-doped GaAs with the concentration of the order of $2 \cdot 10^{11}$ to $3 \cdot 10^{11} \mathrm{~cm}^{-2}$ $[10,11]$ the states of impurities form an impurity band. The conducting states below the conduction band bottom appear. With an increase of the concentration, the impurity band widens and at last overlaps with the 2D conduction band. Furthermore, as mentioned in many papers (for example, [11]), one should take into account that, because of fluctuations of the distances between impurity atoms in the delta-layer, the impurity band widens even more, and the edge of the band (2D subband) becomes smeared.

In the structures doped in a barrier independently of the impurity concentration (up to $1 \cdot 10^{12} \mathrm{~cm}^{-2}$ ), practically all electrons tend to fall into the quantum well. Scattering of carriers at low temperatures in these samples is mainly caused by remote impurities, roughness of well boundaries and alloy fluctuations in InGaAs. The mobility in this case is comparatively high. At high temperatures, a considerable portion of electrons is transferred into states above the barrier. Then scattering of electrons occurs mainly by lattice vibrations, and mobility decreases down to the bulk value.
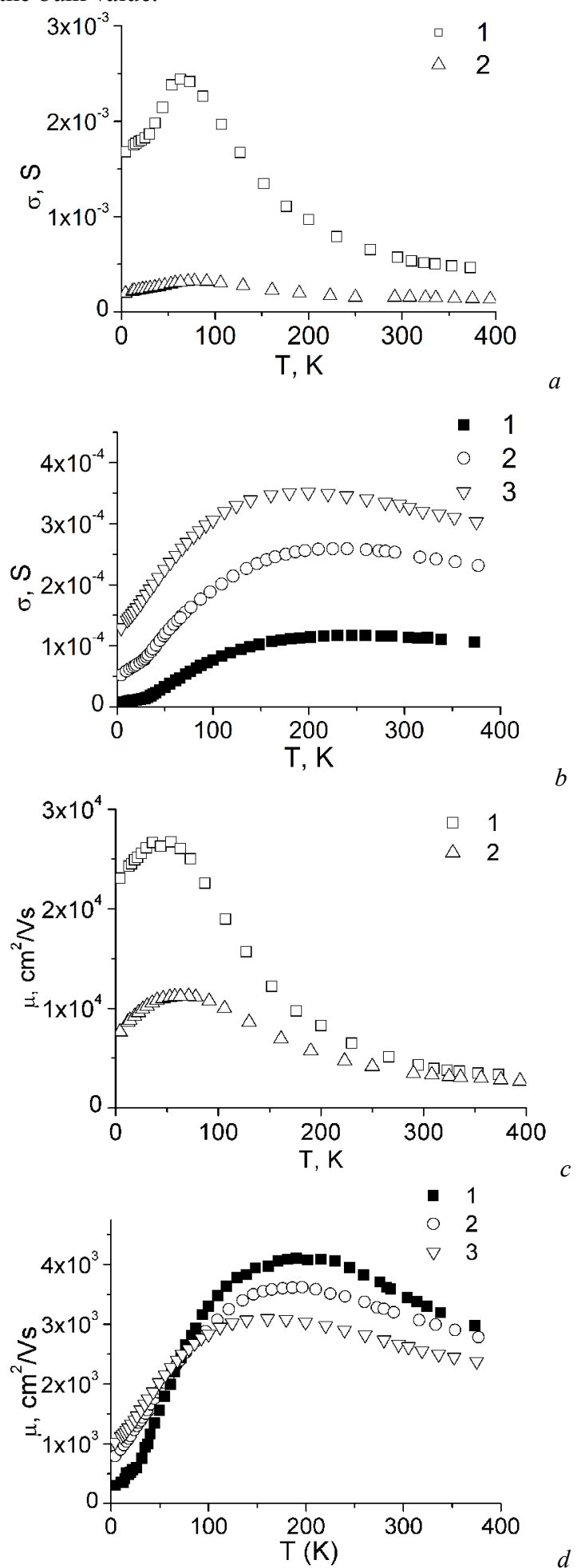

Fig. 2. Temperature dependences of conductivity and mobility of electrons for the samples with the delta-layer of impurity in the barrier $(a$ and $c$ ) and QW centre ( $b$ and $d$ ). Sample \#\# in $a$ and $c$ : $1-6215,2-6291$, in $b$ and $d: 1-5998,2-6002,3-5997$. 
In the structures doped in QW, the situation changes. At low temperatures, if the impurity concentration is lower than that corresponding to overlapping of impurity and conduction bands, the magnitude of mobility is very small. It increases with temperature, because more and more electrons are transferred into 2D subband states. Provided the impurity and conduction bands overlap, then already at low temperatures the conduction is determined by electrons in the states of the 2D subband in QW. However, mobility in this case has a lower value as compared to doping in a barrier because of closeness of the scattering impurity ions to electrons. At the same time, it has a higher value as compared to the low doping concentration when the states do not overlap. The calculations performed in the next section take into account these features of delta-doping in QW.

\section{Numerical calculations}

The aim of this section is, firstly, to construct the model of the potential profile in the studied structures, which is caused by a finite height of the barrier and distribution of the space charge (concentration profile of the ionized impurity and free electrons) along the direction of the heterostructure layers growth, calculation of energy levels and envelope functions of electrons in this potential profile, and, secondly, calculations of mobility in this model. Calculations were carried out for a set of temperatures within the range from 4 to $400 \mathrm{~K}$.

We have used an idealized model of an infinite chain of independent quantum wells and neglected the non-parabolicity of the conduction band. Its influence on the energy levels and on the electron population is less than a few percents even at the highest concentration used in calculations. The positive charge of the impurities in the delta-layer is assumed uniformly distributed over the plane (the jelly model). This assumption is justified in the case of doping in QW, at least if the average distance between the ions is less than a characteristic size of the electron wave function of the impurity in the ground state (for GaAs it corresponds approximately to $N_{\mathrm{S}}>2.5 \cdot 10^{11} \mathrm{~cm}^{-2}$ ).

The system to be solved consists of the onedimensional Schroedinger and Poisson equations supplemented with the electro-neutrality condition,

$$
\begin{aligned}
& -\frac{\hbar^{2}}{2} \frac{d}{d z}\left[\frac{1}{m(z)} \frac{d \psi(z)}{d z}\right]+U(z) \psi(z)=E \psi(z), \\
& \frac{d}{d x}\left[\varepsilon(z) \frac{d U(z)}{d z}\right]=4 \pi e^{2}\left[n(z)-N^{+}(z)\right], \\
& \int_{-L}^{L} N_{D}^{+}(z) d z-\int_{-L}^{L} n(z) d z=0,
\end{aligned}
$$

where $U(z)$ is the coordinate dependence of the energy of the conduction band bottom, $E$ - eigenvalue of the energy, $\psi(z)$ - envelope wave function of an electron along the growth direction of the layers, $m(z), \varepsilon(z), n(z)$ and $N^{+}(z)$ are the coordinate dependences of the electron effective mass, dielectric permittivity, concentration of free electrons and ionized impurities, respectively. Since the quantum wells in the chain are identical, we consider only one period of a heterostructure. The corresponding boundary conditions for one period are written as follows:

$$
\begin{aligned}
& \psi_{n}(L)=\psi_{n}(-L)=0 ; \\
& U(L)-U(-L)=0, \quad \frac{d U(L)}{d z}=\frac{d U(-L)}{d z} .
\end{aligned}
$$

The quantum wells in adjacent periods are separated by wide and non-transparent for electrons barriers where the magnitude of the envelope wave functions exponentially decreases down to zero. The dependences of $\varepsilon(z)$ and $m(z)$ on the coordinate are caused by presence of the quantum wells, where these parameters have values corresponding to InGaAs with a given composition of In.

The concentration of positively charged impurities is determined as

$$
N^{+}(z)=\frac{N(z)}{1+g \exp \left(\frac{E_{\mathrm{F}}-E_{\text {imp }}(z)}{k T}\right)},
$$

where $N(z)=N_{\text {delta }}(z)+N_{b g}(z)$ is the coordinate distribution of the impurity, $g$ - degeneracy factor of the impurity level. The delta-doped impurity concentration is determined by the Gauss distribution function

$$
N_{\text {delta }}(z)=N_{\text {delta }} \frac{1}{\sqrt{2 \pi} \sigma} \exp \left(-\frac{\left(z-z_{0}\right)^{2}}{2 \sigma^{2}}\right) .
$$

Here, $N_{b g}$ is the background impurity, $E_{\mathrm{F}}-$ Fermi level, $E_{\text {imp }}(z)$ - energy of the impurity level in the point $z$ (different in QW and barriers). The concentration of free electrons $n(z)$ is determined in each point as the sum of the concentrations in states above the barrier, which we consider as 3D states, and in all subbands in quantum wells (2D electron gas).

$$
\begin{aligned}
& n(z)=n_{3 D}(z)+\sum_{i} n_{2 D}(z)=N_{C}^{3 D} F_{1 / 2}\left(E_{\mathrm{F}}-U_{c}(z)\right)+ \\
& +\sum_{i}\left|\Psi_{i}(z)\right|^{2} N_{C}^{2 D} \ln \left(1+\exp \frac{E_{\mathrm{F}}-E_{C i}}{k T}\right),
\end{aligned}
$$

where $N_{C}^{3 D}, N_{C}^{2 D}$ are the effective densities of states in the conduction band for the $3 \mathrm{D}$ and $2 \mathrm{D}$ electron gas, $F_{1 / 2}\left(E_{\mathrm{F}}-U_{C}(z)\right)$ is the Fermi-Dirac integral for the index $1 / 2$.

The parameters of GaAs and InAs used in our calculations are listed in Table 2. The parameters of $\mathrm{In}_{\mathrm{x}} \mathrm{Ga}_{1-\mathrm{x}}$ As were determined by linear interpolation. 
Table 2. Parameters of GaAs and InAs.

\begin{tabular}{|l|c|c|}
\hline \multicolumn{1}{|c|}{ Parameter } & InAs & $\mathrm{GaAs}$ \\
\hline Sound velocity, cm/s & $4.28 \cdot 10^{5}$ & $5.24 \cdot 10^{5}$ \\
\hline Density, $\mathrm{g} / \mathrm{cm}^{3}$ & 5.68 & 5.32 \\
\hline Lattice parameter, $\AA$ & 6.0583 & 5.65325 \\
\hline Optical phonon energy, meV & 30 & 35 \\
\hline Optical deformation potential, eV & 41 & 41 \\
\hline Effective mass & 0.023 & 0.063 \\
\hline Deformation potential, eV & -5.04 & -11 \\
\hline Static dielectric permittivity & 15.15 & 12.9 \\
\hline HF dielectric permittivity & 12.3 & 10.89 \\
\hline
\end{tabular}

The system (1)-(3) was solved self-consistently using numerical methods for various temperatures and impurity concentrations. The equations (1) and (2) were written in the finite-difference form for 1000 points along the length of one period, and the solution was found by a standard procedure for this approach.

Calculations were performed up to distances of $\pm 500 \AA$ from the centre of QW corresponding to the period of our structures. The interval between adjacent points, where the potential and wave functions were calculated, was equal to $1 \AA$. The self-consistent calculations were repeated until the difference between two successive corrections to the potential becomes less than a given value (less than $1 \%$, which provided the neutrality condition to be fulfilled with an accuracy no worse than $1 \cdot 10^{-4}$ ).

The obtained data set for the energy spectrum, energy of the Fermi level and envelope wave functions were used in the next step for calculations of mobility. In heterostructures based on III-V compounds, the main mechanisms of electron scattering are scattering by ionized impurities, polar optical phonons, interfaces roughness, composition fluctuations (alloy scattering). Though it is known that in these compounds the scattering by acoustic and deformation potential optical phonons do not play any substantial role, we included them into consideration for completeness of description. The corresponding expressions for the relaxation time, Hall coefficient and mobility are given in the Appendix. It should be noticed that for electron scattering by polar optical phonons one cannot strictly introduce the momentum relaxation time. Nevertheless, following [12] we used for this scattering mechanism the expression for the time of the average loss of the momentum

$\frac{1}{\tau}=\int \frac{q \cos \Theta}{k} W\left(\bar{k}, \overline{k^{\prime}}\right) d \overline{k^{\prime}}, q=\left|\bar{k}-\overline{k^{\prime}}\right|$,

$\Theta$ being the angle between $\mathbf{q}$ and $\mathbf{k}^{\prime}$.

\section{Results and discussion}

Fig. 3 illustrates the profile of the conduction band bottom for one period in the chain of the quantum wells with parameters of the sample \#5997 and delta-doped in the centre of QW. In all our calculations, we also took into account background impurities with the concentration of $5 \cdot 10^{15} \mathrm{~cm}^{-3}$, which were assumed to be uniformly distributed over a period. The energy of quantum levels, the Fermi level and squared envelope wave functions are also shown in Fig. 3.
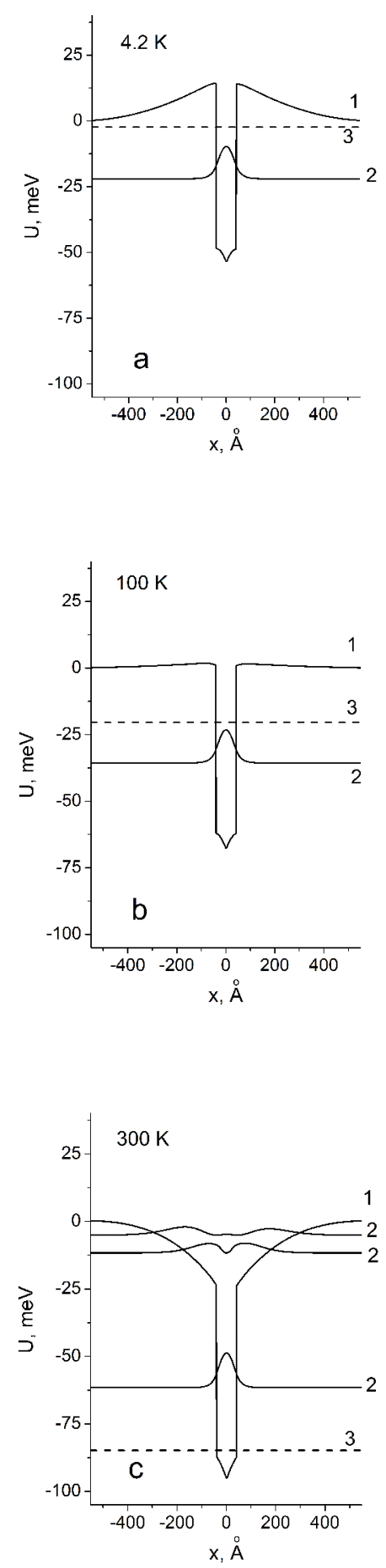

Fig. 3. Energy spectrum in the conduction band for the samples doped by a delta layer of impurity in the centre of QW. The width and depth of QW and impurity concentration correspond to parameters of the sample \#5997. 1 - the conduction band bottom; 2 - square of envelope wave function; 3 - Fermi level. 

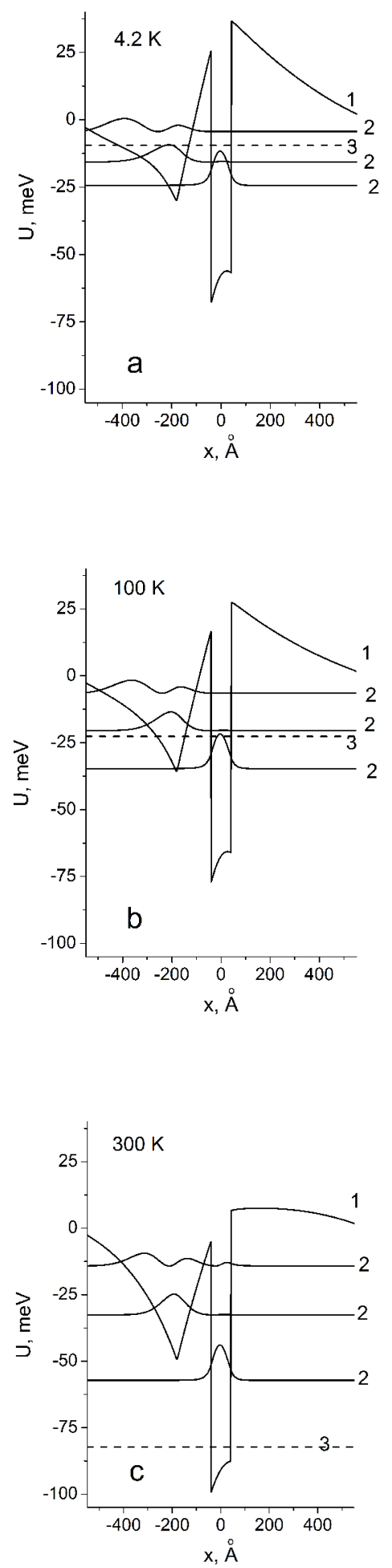

Fig. 4. Energy spectrum in the conduction band for the samples doped by a delta layer of impurity in the barrier. The width and depth of QW and impurity concentration as well as its position correspond to parameters of the sample \#6215. 1 - the conduction band bottom; 2 - square of envelope wave function; 3 - Fermi level.
At low temperature (Fig. 3a), electrons from the background impurities in the barrier are transferred into the lowest energy states (states in QW) and the remaining positive charge causes a rise of the electron potential energy towards the well. Since the electrons from the delta-doping remain in $\mathrm{QW}$, the potential profile weakly depends on this kind of impurity. With temperature growth, electrons come back into the barrier and partially neutralize the positive charge. Approximately at $100 \mathrm{~K}$ (Fig. 3b) all donors in the barrier are neutralized, and the potential profile corresponds to that of rectangular QW. With further temperature growth, electrons of donors located in the central plane of QW begin to penetrate into the barrier. The potential energy related with electric field of the remaining positively charged donors increases with growing the distance from this plane (Fig. 3c). The higher is temperature, the wider and deeper becomes a quantum well. At last, the width of the quantum well becomes comparable with the period of the structure.

Fig. 4 illustrates similar dependences for the structure doped in the barrier with parameters of the sample \#6215. Here, the impurity delta-layer forms the second QW in the barrier with new quantum levels. The profile of the potential energy becomes more complicated, and it does not change qualitatively with temperature growth.

Further it should be noticed that, for structures delta-doped both in the centre of QW (Fig. 3) and in the barrier (Fig. 4) the envelope wave function and, consequently, the electron density do not vanish at the well borders and penetrate quite deep into the barrier. This effect was usually neglected in earlier calculations of low temperature electron mobility in quantum wells $[7,13,14]$; that may be justified in the case of a deep QW or in the case of a large effective mass of charge carriers (for example, in the case of holes in the quantum well of the valence band [15]). Otherwise as shown, for example, in [16] for the interface roughness scattering mechanism, neglecting this factor may cause a significant error in the calculated mobility.

The results obtained by the method described above for a set of temperature values within the range from 4 to $400 \mathrm{~K}$ were used in calculations of the temperature dependence of the mobility. Here, the following two approximations were made, which substantially influenced the obtained results. Firstly, according to the qualitative analysis carried out above, for the structures doped in QW, we assumed a spread of the impurity levels with respect to energy in a shape of the Gauss distribution and put additionally the states lying below the bottom of the first conduction subband in the neutrality equation. This leads to noticeable lowering the Fermi level and considerable decreasing the calculated mobility. Secondly, concerning the mobility in those 2D conduction subbands, which bottoms lie below the states above the barrier by value less than $k T / 4$, it was assumed that it is close to the electron mobility in the 3D states. Therefore, in calculations of the mobility averaged over all bands the 
concentration in these bands was determined with the 2D density of states, while relaxation times were calculated using the formulae for 3D states. Besides, changes in the electron concentration with temperature were neglected in the calculations, and concentration was taken equal to the value measured at $4 \mathrm{~K}$, because in those samples for which the concentration increases considerably by temperatures higher than $100 \mathrm{~K}$ the impurity scattering does not play a noticeable role.

The parameters characterizing scattering processes and used in our calculations are listed in Table 3.

Figs $5 \mathrm{a}$ and $5 \mathrm{~b}$ show the temperature dependences of the electron quasi-momentum relaxation time averaged over the distribution function in the lowest subband for all the scattering mechanisms taken into account. Figs $5 \mathrm{a}$ and $5 \mathrm{~b}$ correspond to delta-doping in the centre of QW and the barrier, respectively.

Table 3. Parameters of the roughness and alloy scattering.

\begin{tabular}{|l|c|}
\hline Roughness correlation length, $\AA$ & 100 \\
\hline Average roughness height, $\AA$ & 2.3 \\
\hline Scattering potential of alloy atoms, eV & 0.33 \\
\hline
\end{tabular}
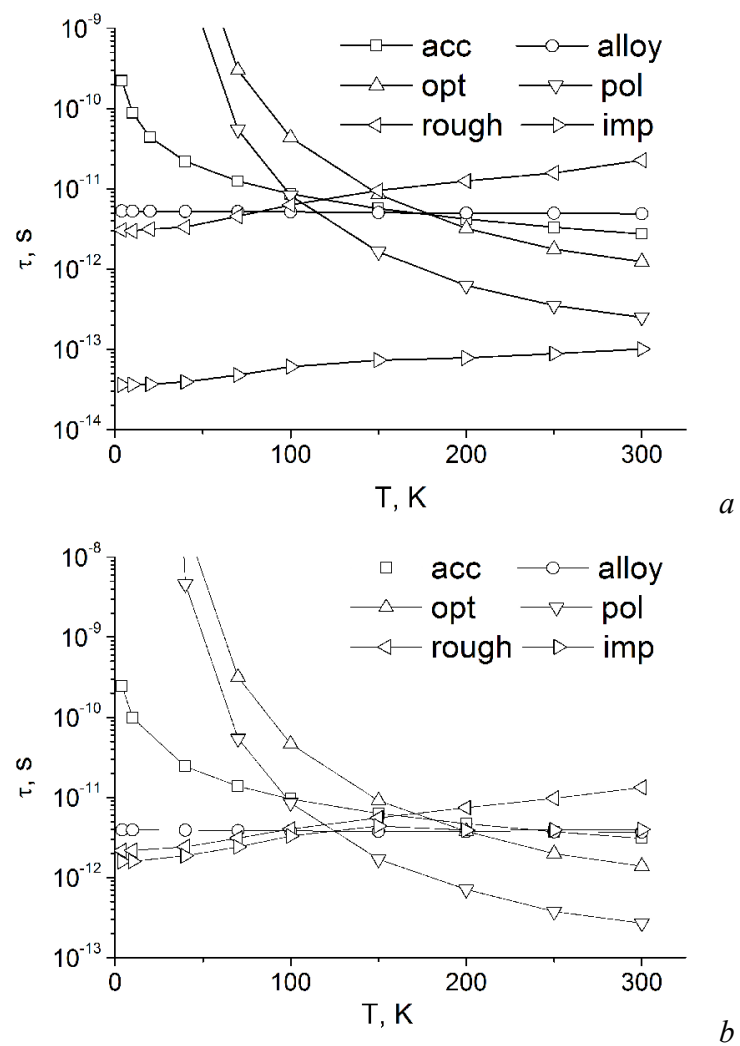

Fig. 5. Temperature dependences of the averaged quasimomentum relaxation times of charge carriers in the lowest $2 \mathrm{D}$ subband in the samples doped by a delta layer of impurity in the centre of QW $(a)$ and barrier $(b)$. Scattering mechanisms: 1 - acoustic phonons; 2 - alloy; 3 - optical deformation potential phonons; 4 - polar optical phonons; 5 - roughness; 6 - impurity.

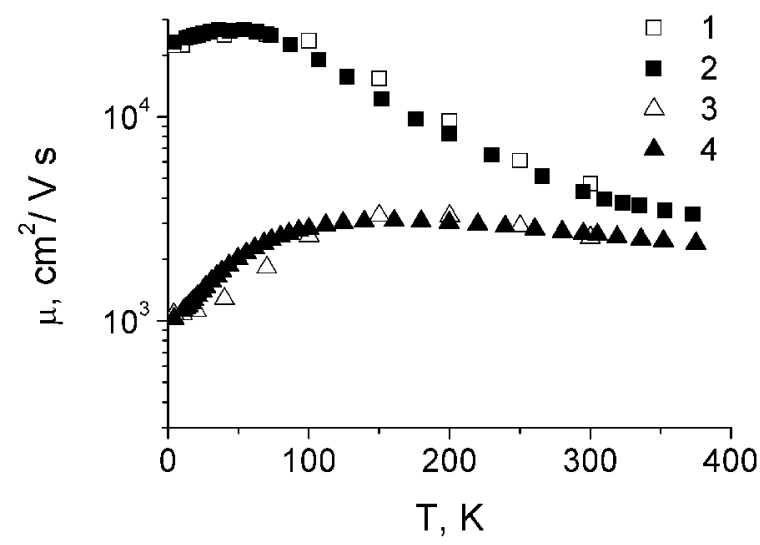

Fig. 6. Fitting the measured temperature dependences of the Hall mobility of electrons in the samples doped by a delta layer of impurity in the barrier $(1,2)$ and in the centre of QW $(3,4)$. 1 and 3 - calculations; 2 and 4 - experiment.

As to be seen, in the case of delta-doping in the centre of QW the prevailing scattering mechanisms are scattering by charged impurities at low temperatures and polar optical phonons at the high ones. For the structures doped in the barrier, a considerable contribution additionally results from scattering on the interface roughness and composition fluctuations at low temperatures and deformation potential optical phonons at high temperatures. The calculated mobilities for samples \#5997 and 6215 are compared with experiment in Fig. 6. As to be seen, all the qualitative features of experimental curves considered above are correctly described by the calculated data. Taking into account the adopted approximations, we conclude that a satisfactory quantitative agreement between measured and calculated data is also achieved. Regarding the other investigated samples a sufficient agreement between experimental and calculated results is also obtained.

\section{Conclusions}

For successful applications of the GaAs/InGaAs/GaAs heterostructures in devices based on the mechanism of a real-space transfer of electrons between tunnelcoupled quantum wells, one must provide a large difference of the mobilities in coupled wells. As seen in Fig. 6, the ratio of mobilities for wells with doping either in the barrier or in one of coupled QW may achieve 20 and even more at $4 \mathrm{~K}$. And this value quickly decreases with temperature growth. However, one should take into account that the dependences of mobility on lattice temperature in equilibrium and on electron temperature under heating electric fields differ in the region where scattering by phonons plays a significant role. Although this ratio should decrease less sharply with growth of electron temperature, the demands to parameters of a structure destined to be used in applications may remain sufficiently rigid, and 
in order to obtain optimal structures, preliminary calculations of their transport properties are needed. A quite good agreement between calculated and experimental dependences obtained in the present work enables to perform sufficiently real calculations of parameters for structures with different applied destination.

\section{Acknowledgments}

The work was supported by State Target Scientific and Technical Program ("Nanotechnologies and Nanomaterials", Project \#1.1.7.18). The authors are also grateful to Prof. O.G. Sarbey and Dr. Sc.V.N. Poroshin for helpful discussion.

\section{Appendix}

Given below are expressions for the quasi-momentum relaxation time used in calculations.

\section{Acoustic phonons}

$\frac{1}{\tau_{a c}(E)}=\frac{m_{0} m_{\text {band }} D_{\text {band }}^{2} k T}{2 \pi \hbar^{3} \rho_{\text {band }} C_{\text {sound }}^{2}} F F_{a c}$,

$F F_{a c}=\int_{-\infty}^{\infty} J^{+} J^{-} d q ; J^{+}=\int|\psi(z)|^{2} \exp \left(i q_{z} z\right) d z$,

$J^{-}=\int|\psi(z)|^{2} \exp \left(-i q_{z} z\right) d z$

$\frac{1}{m_{\text {band }}}=\int \frac{\left|\psi_{\text {band }}(z)\right|^{2} d z}{m(z)}$,

$m_{\text {band }}$ and $m_{0}$ are the effective and free electron masses, $k$ is the Boltzmann constant, $D_{b a n d}, \psi_{\text {band }}, C_{\text {band }}$ are the deformation potential constant, density and sound velocity.

\section{Optical deformation potential phonons}

$\frac{1}{\tau_{o p t}(E)}=\operatorname{Pr} e F \cdot F F_{a c}, \quad E<\hbar \omega_{o p t}$,

$\frac{1}{\tau_{o p t}(E)}=\operatorname{Pr} e F\left[1+\exp \left(\frac{\hbar \omega_{o p t}}{k T}\right)\right] F F_{a c}, E \geq \hbar \omega_{o p t}$,

$\operatorname{Pr} e F=\frac{D_{o p t}^{2} m_{0} m_{\text {band }}}{4 \pi a_{L}^{2} \rho_{\text {band }} \hbar \omega_{o p t} \hbar} \frac{1}{\exp \left(\frac{\hbar \omega_{o p t}}{k T}\right)-1}$,

$a_{L}$ is the lattice parameter.

\section{Polar optical phonons}

$\frac{1}{\tau_{0}}=\frac{e^{2} m_{0} m_{\text {band }} \hbar \omega_{\text {opt }}}{2 \pi \hbar^{3}} \frac{1}{\exp \left(\frac{\hbar \omega_{o p t}}{k T}\right)-1} \times$

$\times \int\left|\psi_{\text {band }}(z)\right|^{2}\left(\frac{1}{\varepsilon_{\infty}(z)}-\frac{1}{\varepsilon_{0}(z)}\right) d z$,

$E<\hbar \omega_{\text {opt }}$,

$F F_{\text {pol }}^{1}=$

$=\int_{-\infty}^{\infty} \frac{\left.\left|\int\right| \psi(z)\right|^{2} \exp (+i q z) d z|| \int|\psi(z)|^{2} \exp (-i q z) d z \mid}{\sqrt{\left(q^{2}+\frac{2 m_{0} m_{\text {band }}}{\hbar^{2} \sqrt{E\left(E+\hbar \omega_{\text {opt }}\right)}}\right)^{2}-\left(\frac{4 m_{0} m_{\text {band }}}{\hbar^{2} \sqrt{E\left(E+\hbar \omega_{\text {opt }}\right)}}\right)^{2}}} d q$,

$q$ is the phonon wavevector participating in scattering

$\frac{1}{\tau(E)}=\frac{1}{\tau_{1}(E)}=\frac{1}{\tau_{0}(E)} \cdot F F_{p o l}^{1}$,

$E \geq \hbar \omega_{o p t}$,

$F F_{\text {pol }}^{\prime \prime}=$

$=\int_{-\infty}^{\infty} \frac{\left.\left|\int\right| \psi(z)\right|^{2} \exp (+i q z) d z|| \int|\psi(z)|^{2} \exp (-i q z) d z \mid}{\sqrt{\left(q^{2}+\frac{2 m_{0} m_{\text {band }}}{\left.\hbar^{2} \sqrt{E\left(E-\hbar \omega_{\text {opt }}\right.}\right)}\right)^{2}-\left(\frac{4 m_{0} m_{\text {band }}}{\hbar^{2} \sqrt{E\left(E-\hbar \omega_{\text {opt }}\right)}}\right)^{2}}} d q$,

$\frac{1}{\tau(E)}=\frac{1}{\tau_{1}(E)}+\frac{2 \pi}{\tau_{0}(E)} \cdot F F_{p o l}^{\prime \prime} \exp \left(\frac{\hbar \omega_{o p t}}{k T}\right)$.

\section{Alloy scattering}

$\frac{1}{\tau_{a l}(E)}=\frac{U_{a l}^{2} a_{\text {lat }}^{3} x(1-x) m_{0} m_{b a n d}}{\hbar^{3}} F F_{a l}$,

$U_{a l}$ is the scattering potential of alloy atoms.

$F F_{a l}=\int_{0}^{L}|\psi(z)|^{4} d z$

\section{Interface roughness scattering}

$\frac{1}{\tau_{\text {rough }}(E)}=\frac{2 m_{0} m_{\text {band }} \pi}{\hbar^{3}} \times$

$\times\left[\left(\frac{(\lambda \Delta)_{\text {left }}^{2}+(\lambda \Delta)_{\text {right }}^{2}}{2}\right)\left(\frac{d E_{C}}{d L}\right)^{2}\right]_{\text {band }} 0 \int_{0}^{1} \frac{\exp \left(-\lambda^{2} k_{0}^{2} x^{2}\right) x^{4} d x}{\sqrt{1-x^{2}}\left(x+x_{S}\right)^{2}}$,

$k_{0}=\sqrt{\frac{2 m_{0} m_{\text {band }} E}{\hbar^{2}}}, x_{S}=\frac{m_{0} m_{\text {band }} e^{2}}{\varepsilon_{0} \hbar^{2} k_{0}}$, 
$\lambda, \Delta$ are the correlation length and magnitude of roughness, $\frac{d E_{C}}{d L}$ is the calculated subband bottom fluctuation with the roughness-caused fluctuation of a well width.

\section{Impurity scattering}

$\frac{1}{\tau(E)}=\frac{4 \pi m e^{4}}{\hbar^{3}} \times$

$\times \int_{0}^{\pi} d \Theta(1-\cos \Theta) \frac{1}{[q \varepsilon(q)]^{2}} \int d z|F(q, z)|^{2} N^{+}(z)$,

$q=2 \sin \frac{\Theta}{2} k_{0}$,

$F(q, z)=\int d z^{\prime}\left|\psi\left(z^{\prime}\right)\right|^{2} \exp \left(-q\left|z-z^{\prime}\right|\right)$,

$\varepsilon(q)=\varepsilon_{0}\left[1+\frac{2 \pi e^{2}}{\varepsilon_{0}} \frac{2 m}{2 \pi \hbar^{2}} F(q)\right]=\varepsilon_{0}+\frac{2 m e^{2}}{\hbar^{2}} F(q)$,

$F(q)=\int d z|\psi(z)|^{2} F(q, z)$.

The total relaxation time for each subband

$$
\begin{aligned}
& \frac{1}{\tau(E)}=\frac{1}{\tau_{a c}(E)}+\frac{1}{\tau_{\text {opt }}(E)}+\frac{1}{\tau_{\text {pol }}(E)}+ \\
& +\frac{1}{\tau_{\text {rough }}(E)}+\frac{1}{\tau_{\text {imp }}(E)}+\frac{1}{\tau_{\text {alloy }}(E)} .
\end{aligned}
$$

For each subband:

$\langle\tau\rangle=\frac{\int E \tau(E) \frac{d f}{d E} d E}{\int f(E) d E}=\frac{1}{4 I_{\text {norm }}} \int_{E_{C}^{\text {band }}}^{0} \frac{E \tau(E) d E}{\left[\operatorname{ch}\left(\frac{E-E_{F}}{2 k T}\right)\right]^{2}}$,

$I_{\text {norm }}=\int_{E_{C}^{\text {band }}}^{0} \frac{d E}{1+\exp \left(\frac{E-E_{F}}{k T}\right)}$,

$\mu=\frac{e}{m_{0} m_{\text {band }}}\langle\tau\rangle$.

The total drift mobility is determined as follows

$\mu_{\text {drift }}=\frac{\sum_{\text {bands }} \mu_{\text {band }} n_{\text {band }}}{\sum_{\text {bands }} n_{\text {band }}}$,

$n_{\text {band }}$ is the electron concentration in a given subband

$n_{\text {band }}=\frac{m_{0} m_{\text {band }} k T}{\pi \hbar^{2}} \times$

$\times \ln \left[1+\exp \left(\frac{E_{F}-E_{\text {Cband }}}{k T}\right)\right]$.
The total Hall mobility is determined as

$\mu_{\mathrm{H}}=\frac{\sum_{\text {bands }} r_{\mathrm{H}} \mu_{\text {band }}^{2} n_{\text {band }}}{\sum_{\text {bands }} \mu_{\text {band }} n_{\text {band }}}$, where $r_{\mathrm{H}}=\frac{\left\langle\tau^{2}\right\rangle}{\langle\tau\rangle^{2}}$.

The quantum wells in the investigated structures are not deep (less than $100 \mathrm{meV}$ ). Consequently, at high temperatures from 200 to $400 \mathrm{~K}$, there are a lot of electrons in the $3 \mathrm{D}$ states that give a noticeable contribution to the measured Hall concentration and mobility. Participating in scattering of 3D electrons are acoustic, optical deformation and polar phonons and ionized impurity. The expressions for 3D electron mobility at these scattering mechanisms are well known and are not given here. They are included in the total mobility as follows

$\mu_{\text {drift }}=\frac{\sum_{\text {bands }} \mu_{\text {band }} n_{\text {band }}+\mu_{3 D} n_{3 D}}{\sum_{\text {bands }} n_{\text {band }}+n_{3 D}}$,

$\mu_{\mathrm{H}}=\frac{\sum_{\text {bands }} r_{\mathrm{H}} \mu_{\text {band }}^{2} n_{\text {band }}+r_{\mathrm{H}}^{3 D} \mu_{3 D}^{2} n_{3 D}}{\sum_{\text {bands }} \mu_{\text {band }} n_{\text {band }}+\mu_{3 D} n_{3 D}}$,

where $n_{3 \mathrm{D}}$ is the integral of the above-barrier (3D) electron concentration within one period.

References

1. Y. Ando, Field Effect Transistors, US Patent 5371387 (1994); C. Chang, GaAs-InGaAs high electron mobility transistor, US Patent 5653440 (1995).

2. F. Bugge, G. Erbert, J. Fricke, S. Gramlich, R. Staske, H. Wenzel, U. Zeimer, and M. Weyers, $12 \mathrm{~W}$ continuous-wave diode lasers at $1120 \mathrm{~nm}$ with InGaAs quantum well // Appl. Phys. Lett. 79, p. 1965-1967 (2001).

3. V.Ya. Aleshkin, A.A. Andronov, A.V. Antonov et al., Toward far- and mid-IR intraband lasers based on hot carrier intervalley/real-space transfer in multiple quantum well systems // SPIE Proc. 4318, p. 192-203 (2001).

4. P.A. Belevski, V.V. Vainberg, M.N. Vinoslavskii, A.V. Kravchenko, V.N. Poroshin, and O.G. Sarbey, Real-space transfer and far-infrared emission of hot electrons in $\mathrm{InGaAs} / \mathrm{GaAs}$ heterostructures with tunnel-coupled quantum wells // Ukr. J. Phys. 54(12), p. 117-122 (2009).

5. N.V. Baidus, P.A. Belevskii, A.A. Biriukov, V.V. Vainberg, M.N. Vinoslavskii, A.V. Ikonnikov, B.N. Zvonkov, A.S. Pylypchuk and V.N. Poroshin, Lateral transport and far-infrared radiation of electrons in $\mathrm{In}_{\mathrm{x}} \mathrm{Ga}_{1-\mathrm{x}} \mathrm{As} / \mathrm{GaAs}$ heterostructures with the double tunnel-coupled quantum wells in a high electric field // Semiconductors, 44, p. 1495-1498 (2010). 
6. W. Ted. Masselink, High-differential mobility of hot electrons in delta-doped quantum wells // Appl. Phys. Lett. 59, p. 694-696 (1991).

7. W. Ted. Masselink, Ionized-impurity scattering of quasi-two-dimensional quantum-confined carriers // Phys. Rev. Lett. 66(11), p. 1513-1516 (1991).

8. Il-Ho Ahn, G.Hugh Song, Young-Dahl Jho, Separating the contribution of mobility among different quantum well subbands // Jpn. J. Appl. Phys. 49, 014102-014105 (2010).

9. V.A. Kulbachinskii, I.S. Vasil'evskii, R.A. Lunin, G. Galistu, Electron transport and optical properties of shallow $\mathrm{GaAs} / \mathrm{InGaAs} / \mathrm{GaAs}$ quantum wells with a thin central AlAs barrier // Semicond. Sci. Technol. 22, p. 222-228 (2007).

10. T. Ando, A.B. Fowler, F. Stern, Electronic properties of two-dimensional systems // Reviews of Modern Physics, 54 (2), p. 437-672 (1982).

11. Gold A. Gold, A. Ghazali, J. Serre, Electronic properties of $\delta$-doped GaAs // Semicond. Sci. Technol. 7, p. 972-979 (1992).
12. B.K. Ridley, The electron-phonon interaction in quasi-twodimensional semiconductor quantum-well structures // J. Phys. C: Solid State Phys., 15, p. $5899-5917$ (1982).

13. J. Lee, H.N. Spector, V.K. Arora, Impurity scattering limited mobility in a quantum well heterojunction // J. Appl. Phys. 54(12), p. 6995 7004 (1983).

14. G. Fishman and D. Calecki, Electron concentration and buffer-width dependence of Hall mobility in GaAs-GaAlAs multiple-quantum-well structures // Phys. Rev. B, 29, p. 5778-5787 (1984).

15. B. Laikhtman, R.A. Kiehl, Theoretical hole mobility in a narrow $\mathrm{Si} / \mathrm{SiGe}$ quantum well // Phys.Rev. B, 47, p. 10515-10527 (1993).

16. J.M. Li, J.J. Wu, X.X. Han, Y.W. Lu, X.L. Liu, Q.S. Zhu and Z.G. Wang, A model for scattering due to interface roughness in finite quantum wells // Semicond. Sci. Technol. 20, p. 1207-1212 (2005). 